\title{
The SABRE project and the SABRE Proof-of-Principle
}

\author{
M. Antonello ${ }^{1}$, E. Barberio ${ }^{2}$, T. Baroncelli ${ }^{2}$, J. Benziger ${ }^{3}$, L. J. Bignell ${ }^{4}$, I. Bolognino ${ }^{1,5}$, F. Calaprice ${ }^{6}$, S. Copello ${ }^{7,8}$, \\ D. D’Angelo ${ }^{1,5}$, G. D'Imperio ${ }^{9}$, a ${ }^{\prime}$ I. Dafinei ${ }^{9}$, G. Di Carlo ${ }^{7}$, M. Diemoz ${ }^{9}$, A. Di Ludovico ${ }^{6}$, W. Dix ${ }^{2}$, A. R. Duffy ${ }^{10,11}$, \\ F. Froborg ${ }^{12}$, G. K. Giovanetti ${ }^{6}$, E. Hoppe ${ }^{13}$, A. Ianni ${ }^{7}$, L. Ioannucci ${ }^{7}$, S. Krishnan ${ }^{11}$, G. J. Lane ${ }^{4}$, I. Mahmood ${ }^{2}$, \\ A. Mariani ${ }^{8}$, M. Mastrodicasa ${ }^{9,14}$, P. Montini ${ }^{9,14,17}$, J. Mould ${ }^{10,11}$, F. Nuti ${ }^{2}$, D. Orlandi ${ }^{7}$, M. Paris ${ }^{7}$, V. Pettinacci ${ }^{9}$, \\ L. Pietrofaccia ${ }^{6}$, D. Prokopovic ${ }^{16}$, S. Rahatlou ${ }^{9,14}$, N. Rossi ${ }^{9}$, A. Sarbutt ${ }^{16}$, E. Shields ${ }^{6}$, M. J. Souza ${ }^{6}$,

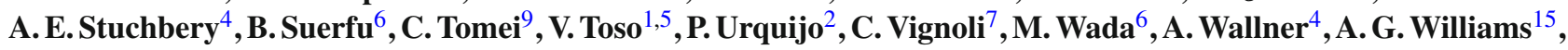 \\ J. $\mathbf{X u}^{6}$ \\ ${ }^{1}$ INFN, Sezione di Milano, 20133 Milan, Italy \\ ${ }^{2}$ School of Physics, The University of Melbourne, Melbourne, VIC 3010, Australia \\ ${ }^{3}$ Chemical Engineering Department, Princeton University, Princeton, NJ 08544, USA \\ ${ }^{4}$ Department of Nuclear Physics, The Australian National University, Canberra, ACT 2601, Australia \\ ${ }^{5}$ Dipartimento di Fisica, Università degli Studi di Milano, 20133 Milan, Italy \\ ${ }^{6}$ Physics Department, Princeton University, Princeton, NJ 08544, USA \\ ${ }^{7}$ INFN, Laboratori Nazionali del Gran Sasso, 67100 Assergi (L’Aquila), Italy \\ ${ }^{8}$ INFN, Gran Sasso Science Institute, 67100 L'Aquila, Italy \\ ${ }^{9}$ INFN, Sezione di Roma, 00185 Rome, Italy \\ ${ }^{10}$ ARC Centre of Excellence for All-Sky Astrophysics (CAASTRO), Sydney, Australia \\ ${ }^{11}$ Centre for Astrophysics and Supercomputing, Swinburne University of Technology, PO Box 218, Hawthorn, VIC 3122, Australia \\ 12 High Energy Physics, Blackett Laboratory, Imperial College London, London SW7 2BZ, UK \\ ${ }^{13}$ Pacific Northwest National Laboratory, 902 Battelle Boulevard, Richland, WA 99352, USA \\ ${ }^{14}$ Dipartimento di Fisica, Sapienza Università di Roma, 00185 Rome, Italy \\ 15 The University of Adelaide, Adelaide, SA 5005, Australia \\ ${ }^{16}$ Australian Nuclear Science and Technology Organization, Lucas Heights, NSW 2234, Australia \\ 17 Present address: Dipartimento di Matematica e Fisica Università di Roma Tre and INFN Sezione di Roma Tre, 00146 Rome, Italy
}

Received: 15 November 2018 / Accepted: 5 April 2019 / Published online: 26 April 2019

(C) The Author(s) 2019

\begin{abstract}
SABRE aims to directly measure the annual modulation of the dark matter interaction rate with $\mathrm{NaI}(\mathrm{Tl})$ crystals. A modulation compatible with the standard hypothesis, in which our Galaxy is immersed in a dark matter halo, has been measured by the DAMA experiment in the same target material. Other direct detection experiments, using different target materials, seem to exclude the interpretation of such modulation in the simplest scenario of WIMP-nucleon elastic scattering. The SABRE experiment aims to carry out an independent search with sufficient sensitivity to confirm or refute the DAMA claim. The goal of the SABRE experiment is to achieve the lowest background rate for a $\mathrm{NaI}(\mathrm{Tl})$ experiment (order of $0.1 \mathrm{cpd} / \mathrm{kg} / \mathrm{keV}_{\text {ee }}$ in the energy region of interest for dark matter). This challenging goal could be achievable by operating high-purity crystals inside a liquid scintillator veto for active background rejection. In addition, twin detectors will be located in the northern and southern hemispheres to identify possible contributions to the modula-
\end{abstract}

a e-mail: giulia.dimperio@ roma1.infn.it tion from seasonal or site-related effects. The SABRE project includes an initial Proof-of-Principle phase at LNGS (Italy), to assess the radio-purity of the crystals and the efficiency of the liquid scintillator veto. This paper describes the general concept of SABRE and the expected sensitivity to WIMP annual modulation.

\section{Motivation}

For decades, direct detection experiments have been searching for interactions of dark matter candidates with Standard Model particles. A net flux of dark matter through terrestrial detectors is expected assuming that the solar system moves through a dispersion dominated dark matter halo surrounding our galaxy. Due to the Earth's orbital motion around the Sun, the predicted interaction rate of dark matter in a target material undergoes an annual modulation with a characteristic phase [1,2]. The DAMA experiment (short for DAMA/NaI and DAMA/LIBRA) has observed a clear annual modula- 
tion exploiting $\mathrm{NaI}(\mathrm{Tl})$ crystals at Laboratori Nazionali del Gran Sasso (LNGS) in Italy. This is a model-independent finding which satisfies the criteria for a WIMP induced signal [3]. Recently, the DAMA collaboration has released the first results from their Phase-2 experiment [4], confirming the evidence of a signal that meets all the requirements of a model-independent dark matter annual modulation signature at $12.9 \sigma$ significance. Results from several other experiments, when interpreted in the standard WIMP galactic halo hypothesis, seem to exclude the interpretation of the DAMA signal as due to spin-independent WIMPs nuclear scattering. Currently, the best sensitivity in the mass region above $10 \mathrm{GeV} / \mathrm{c}^{2}$ is reported by the XENON1T experiment [5], while the most stringent results at low energy are set by the Darkside-50 [6], CDMSlite [7], and CRESST-III [8] experiments.

The Sodium-iodide with Active Background REjection (SABRE) experiment aims to perform a measurement of the dark matter annual modulation with $\mathrm{NaI}(\mathrm{Tl})$ crystals, aiming to a lower background than that of the DAMA experiment. The experiment will consist of twin detectors located in both the Northern and Southern hemispheres to disentangle possible seasonal or site effects from the dark matter modulation. The dual site is a unique feature of SABRE, with respect to other $\mathrm{NaI}(\mathrm{Tl})$ dark matter searches currently running: the COSINE-100 experiment [9] at the Yang Yang Laboratory in South Korea and the ANAIS experiment [10] at the Canfranc Laboratory in Spain. These experiments, even after several years of operation, might not be able to resolve all possible scenarios in interpreting the DAMA signal as a dark matter signature, given that their background levels are about 2-3 times higher than that of DAMA. On the other hand, if the modulation is observed by SABRE consistently in both hemispheres, a precise measurement of the low energy recoil spectrum and of the dependence with energy of the modulated amplitude might offer insights on how dark matter particles interact with ordinary matter and perhaps on their density and velocity in the galactic halo [11,12]. The hypothesis of a focusing effect of the dark matter wind due to the gravitational potential of the Sun could also be investigated [13].

\section{The SABRE concept}

The SABRE experiment uses $\mathrm{NaI}(\mathrm{Tl})$ scintillating crystals for dark matter detection and focuses on the achievement of a background of the order of $0.1 \mathrm{cpd} / \mathrm{kg} / \mathrm{keV}_{\mathrm{ee}}{ }^{1}$ at few $\mathrm{keV}_{\mathrm{ee}}$ of energy, that is the range of interest for dark matter searches with $\mathrm{NaI}(\mathrm{Tl})$ detectors.

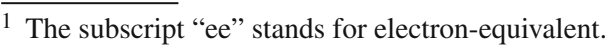

The substantial background reduction anticipated by SABRE, namely one order of magnitude below the level reached by the DAMA experiment and not yet matched by any other $\mathrm{NaI}(\mathrm{Tl})$ experiment to date, could be achieved via ultra-high crystal radiopurity coupled with active rejection through a liquid scintillator veto. Both features will be tested in an initial Proof-of-Principle phase, denoted SABRE-PoP.

The SABRE-PoP setup is presently under construction at LNGS under a rock coverage equivalent to $3600 \mathrm{~m}$ of water. SABRE-PoP is expected to run prior to the deployment of the full-scale experiment. This will consist of two twin detectors: SABRE-North at LNGS and SABRE-South at the Stawell Underground Physics Laboratory (SUPL) in the Southern hemisphere (see Sect. 2.3).

The following sections introduce the main ideas of the SABRE project and describe the Research \& Development carried out so far by the SABRE Collaboration.

\section{1 $\mathrm{NaI}(\mathrm{Tl})$ detectors}

A crucial part of the SABRE effort is the development of high-purity $\mathrm{NaI}(\mathrm{Tl})$ crystals. The background from decays due to radioactive impurities in the crystals is indeed the hardest to suppress. These processes can produce energy signals of a few $\mathrm{keV}_{\mathrm{ee}}$, compatible with the expected recoil energies due to dark matter interactions and within the energy range where DAMA's modulation is observed $\left(2-6 \mathrm{keV}_{\mathrm{ee}}\right.$ [3]). Among the potential background sources, ${ }^{40} \mathrm{~K}$ is the most dangerous since one of its decay channels causes a peak at $\sim 3 \mathrm{keV}$ from Auger/X-ray emissions. Worrisome contributions come from ${ }^{210} \mathrm{~Pb}$ and cosmogenic activated isotopes [14], especially ${ }^{3} \mathrm{H}$. Other contaminants to limit include ${ }^{238} \mathrm{U}$, ${ }^{232} \mathrm{Th},{ }^{87} \mathrm{Rb}$. The background in $\mathrm{NaI}(\mathrm{Tl})$ detectors for dark matter searches has been thoroughly studied $[15,16]$ by the currently running experiments mentioned in Sect. 1. For a study of the background contribution from internal radioactivity of the SABRE crystals we refer to the Monte Carlo simulations performed for the Proof-of-Principle phase [17].

To keep the intrinsic contaminants at a very low level, SABRE has developed a method to obtain ultra-pure NaI powder and a clean procedure to grow crystals. Princeton University and industrial partner Sigma-Aldrich [18] have produced ultra-high purity NaI powder, so-called Astro Grade powder, with potassium levels consistently lower than $10 \mathrm{ppb}$. The content of ${ }^{238} \mathrm{U}$ and ${ }^{232} \mathrm{Th}$ in Astro Grade powder has been measured by Inductively Coupled Plasma Mass Spectrometry (ICP-MS) [19] obtaining for both the isotopes the upper limit of $1 \mathrm{ppt}$. The $\mathrm{NaI}(\mathrm{Tl})$ crystals are then grown by Radiation Monitoring Devices, Inc. (RMD) [20].

RMD company uses the vertical Bridgman-Stockbarger technique [21], where the powder is placed inside a sealed ampoule. The sealed environment reduces the possibility of contamination of the material during the growth phase. To 
determine the best ampoule composition, several materials were prepared with different cleaning procedures. Crystals were grown from Astro Grade powder following these procedures and tested for radioactive contaminations with ICPMS. The optimum ampoule composition, together with a precision cleaning, showed no increase of the impurity levels inside the crystal with respect to the starting powder. At the end of 2015, the optimal procedure was used to grow a 2$\mathrm{kg}$ crystal with average ${ }^{39} \mathrm{~K}$ level of $9 \pm 1 \mathrm{ppb}[19,22,23]$ and with ${ }^{87} \mathrm{Rb}$ upper limit of $0.1 \mathrm{ppb}$ measured by ICP-MS [23]. As a comparison, DAMA reports an average of $13 \mathrm{ppb}$ ${ }^{\text {nat }} \mathrm{K}$ in their crystals (equivalent to $12.1 \mathrm{ppb}$ of ${ }^{39} \mathrm{~K}$ ), ${ }^{238} \mathrm{U}$ and ${ }^{232} \mathrm{Th}$ content below $0.01 \mathrm{ppb}$, and an upper limit on the content of ${ }^{87} \mathrm{Rb}(<0.35 \mathrm{ppb})$ [24].

The crystal purity can only be partially evaluated by ICPMS or Accelerator Mass Spectrometry (AMS), a mass spectrometry that involves accelerating the ions to high kinetic energies. Both techniques are only sensitive to primordial parents. Direct counting of the intrinsic radioactivity by operating the crystal as a scintillator is still the most accurate method and can also detect cosmogenic activation that occurred prior to installation of the crystal underground. The SABRE-PoP phase is in preparation at LNGS to assess the SABRE crystal purity by directly operating the crystal as a scintillator inside an active veto in an underground setup. RMD is currently growing cylindrical $\mathrm{NaI}(\mathrm{Tl})$ crystals for a mass of about $5 \mathrm{~kg}$ for each crystal. The first of these crystals will be tested within the PoP setup.

In the SABRE design, two PMTs are directly coupled onto each flat end of the crystal, while its curved side is wrapped with reflector for better light collection efficiency. We anticipate using PMTs with high quantum efficiency, very low background, and low dark counts in order to access energies below $2 \mathrm{keV}_{\text {ee }}$. The detector assembly is placed inside a high-purity, air- and light-tight copper enclosure. The technical design of the detector module developed for the SABREPoP phase is described in Sect. 3.

\subsection{Active background rejection system}

SABRE aims to further reduce the background level by placing an array of detector modules $(\mathrm{NaI}(\mathrm{Tl})$ crystals coupled to PMTs) inside a liquid scintillator veto. This system consists of a vessel filled with scintillating fluid and equipped with PMTs on its walls. Events with simultaneous energy depositions in the veto and the crystal can be identified as background. For instance, the dangerous $3 \mathrm{keV}_{\text {ee }}$ signal following the electron capture of ${ }^{40} \mathrm{~K}$ in the $\mathrm{NaI}(\mathrm{Tl})$ crystal can be rejected by detecting the coincident $1.46 \mathrm{MeV}$ gamma signal in the surrounding material. Experiments such as DAMA and ANAIS [25] can only reject events with coincident energy deposition in neighbouring crystals, achieving a partial suppression of this background. However, the SABRE strategy (adopted also by the COSINE experiment [26]) with $4 \pi$ active volume around the crystal matrix, has the potential to identify and suppress the ${ }^{40} \mathrm{~K}$ background with higher efficiency (expected rejection of $84 \%$ in $2-6 \mathrm{keV}_{\text {ee }}$ region [17]). We anticipate using an organic scintillator mixture based on pseudocumene and high quantum efficiency PMTs. This combination will be tested within the SABRE-PoP setup, as described in Sect. 3. SABRE-South is exploring an alternative liquid scintillator based on linear alkyl benzene (LAB) solvent, due to its less stringent safety handling constraints (higher flash point).

\subsection{Twin detectors}

A unique feature of SABRE is to adopt twin detectors located in the North and South hemispheres. The annual modulation of the experimental rate induced by dark matter interaction is expected to have the same phase in both hemispheres, given its galactic origin. In contrast, an annual modulation of the rate due to seasonal or site effects would be characterized by a different phase and amplitude in the two detectors. The SABRE twin detectors will be placed at LNGS, Italy, and at the Stawell Underground Physics Laboratory (SUPL) in Australia, located $240 \mathrm{~km}$ north-west of Melbourne. A section of an active gold mine is being converted into the laboratory. The chosen site is $1025 \mathrm{~m}$ deep with a flat overburden, corresponding to approximately $3000 \mathrm{~m}$ water equivalent depth, similar to LNGS. SUPL will be the first underground laboratory in the southern hemisphere, and will be operative by the beginning of 2020 .

\section{SABRE-PoP technical design}

The SABRE-PoP phase has the goal of assessing the crystal purity, the effectiveness of the active background rejection system, and the overall background level. This phase will run with a single high-purity cylindrical $\mathrm{NaI}(\mathrm{Tl})$ crystal, for a total mass of $\sim 5-\mathrm{kg}$, which is currently being grown by RMD from Astro Grade powder following the procedure described above. The crystal will be wrapped with Polytetrafluoroethylene (PTFE) as reflector and coupled at each end to 3-inch Hamamatsu R11065-20 PMTs. These PMTs are specifically developed for low-background experiments as they have high quantum efficiency of $30-35 \%$ at $420 \mathrm{~nm}$ and low intrinsic radioactivity [27].

The assembly will be sealed in a copper enclosure, as shown in Fig. 1.

The cylinder, the end-caps and the support rods are made out of high-purity, low-radioactivity, oxygen-free electrolytic copper $\mathrm{C} 10100$. The holders for the crystal and the PMTs are made out of high-purity PTFE. Given that sodium-iodide is highly hygroscopic, all parts will be thermally treated prior 


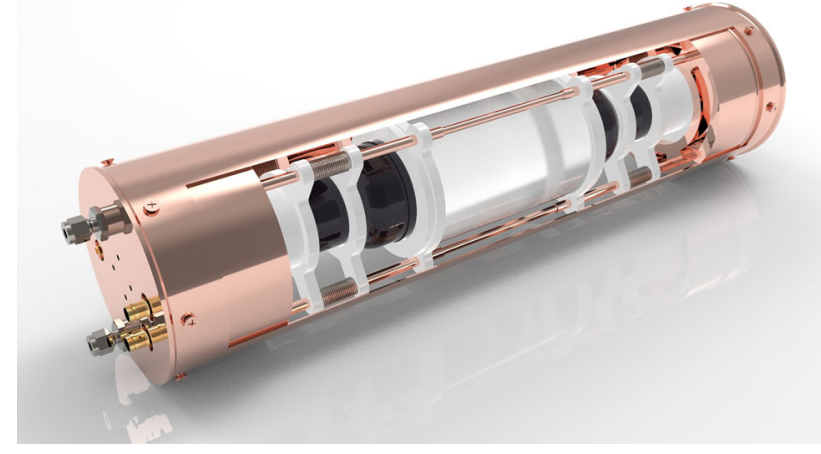

Fig. 1 Rendering of the detector module for the SABRE-PoP phase. The crystal is placed in a copper enclosure; the crystal wrapping is not shown. Two PMTs (black body) are coupled on each end of the crystal. PTFE holders (white) and copper rods keep the crystal and the PMTs in position

to assembly to remove any residual water. After the sealing, the detector module will be flushed with high purity $\mathrm{N}_{2}$ gas to avoid humidity and radon. The cylindrical part of the enclosure has a diameter of $14.6 \mathrm{~cm}$, a height of $58 \mathrm{~cm}$ and a thickness of $2 \mathrm{~mm}$. It is closed by a shaft seal with Viton ${ }^{\circledR}$ O-rings. The enclosure sits vertically and the top end-cap is furnished with bulkhead feedthroughs for the PMT high voltage and signal cables and for teflon tubes. The latter will allow flushing of the inner volume with dry nitrogen gas. The copper enclosure will be placed inside a $1.3 \mathrm{~m}$ (diameter) $\times 1.5 \mathrm{~m}$ (length) cylindrical vessel filled with about two tons of pseudocumene and instrumented with ten 8-inch PMTs. The vessel is made from low-radioactivity stainless steel with access via a $60 \mathrm{~cm}$ diameter flange at the top. The vessel's inner surface is coated with Ethylene tetrafluoroethylene (ETFE) to prevent scintillator degradation due to direct contact with the stainless steel. A Lumirror ${ }^{\mathrm{TM}}$ liner with $95 \%$ reflectance above $400 \mathrm{~nm}$ is applied inside to improve light reflection. Each flat end of the vessel hosts five Hamamatsu R5912-100 PMTs with 35\% quantum efficiency at $390 \mathrm{~nm}$. The liquid scintillator is $(1,2,4)$ Trimethylbenzene (pseudocumene, PC) from the Borexino facility [28], doped with $3 \mathrm{~g} / \mathrm{l}$ of $(2,5)$-diphenyloxazole (PPO), which acts as wavelength shifter. The expected light yield is 0.22 photoelectrons $/ \mathrm{keV}_{\text {ee }}$ [29].

The detector and the fluid handling system design foresee a slow control system that will ensure precise and stable control over all operational parameters such as temperature, pressure, and radon emanation, with the double goal of ensuring security and control of all variables relevant for the physics analysis.

To allow the insertion of the detector module, through the top flange of the vessel, a $2 \mathrm{~mm}$ thick copper tube $(16 \mathrm{~cm}$ diameter and $121 \mathrm{~cm}$ height) is connected to the top cover plate of the vessel and provides a dry volume inside the steel

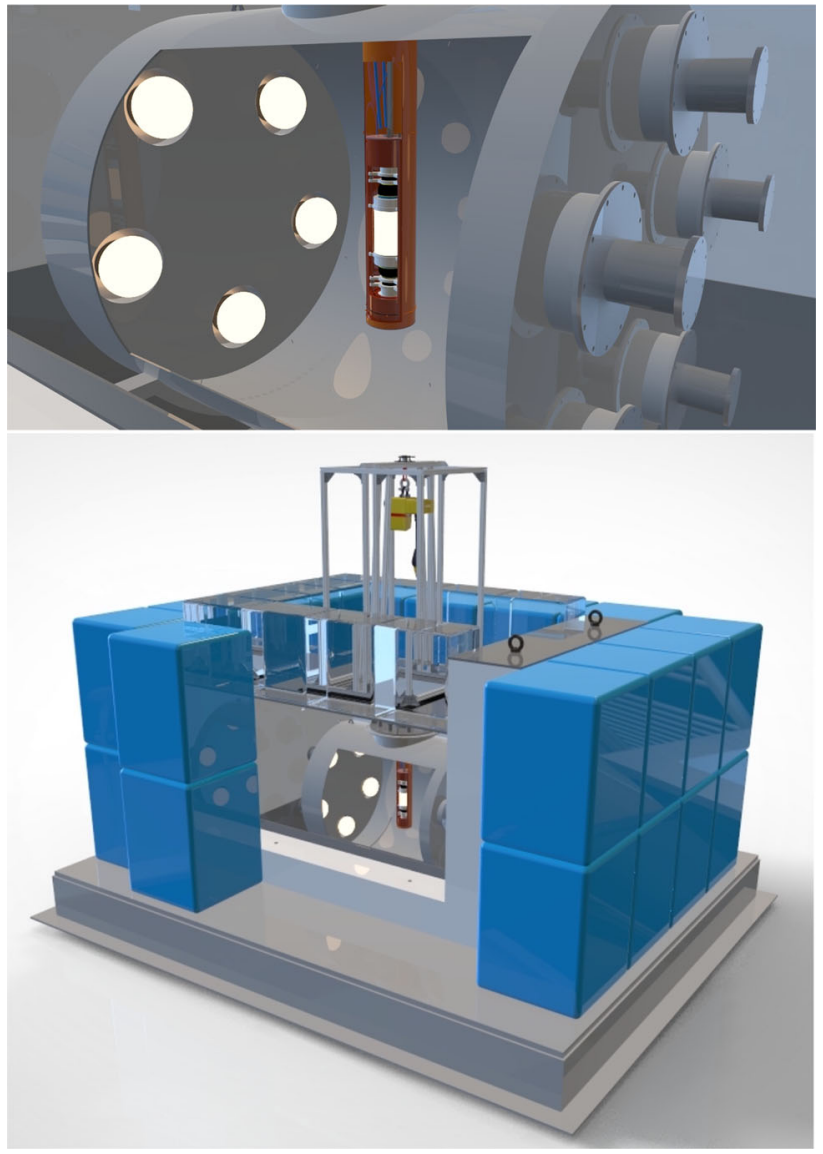

Fig. 2 Top: the SABRE-PoP insertion system. A thin copper tube is connected to the top cover plate of the vessel to provide a dedicated dry volume for the detector module. Bottom: a removable frame is mounted at the top of the vessel to lower the detector module with a motorized pulley into the tube. The vessel is surrounded on each side by polyethylene. On the bottom, the detector is isolated by lead layers, while the sides and the roof are shielded by water tanks

vessel. The detector module will be inserted in this tube without any contact of the scintillator with air or the outside environment during the operation. The inner volume of the tube is flushed with dry and clean $\mathrm{N}_{2}$-gas through teflon tubes running into the small space between the copper tube and the enclosure. This flushing serves as a safety blanket against moisture, radon gas or other background sources. An additional teflon tube allows for the insertion of wire-mounted calibration sources: we plan to use ${ }^{109} \mathrm{Cd},{ }^{57} \mathrm{Co}$, and ${ }^{241} \mathrm{Am}$ for the low energy and ${ }^{228} \mathrm{Th}$ for the high energy region. We will use the reconstructed energy of the $3 \mathrm{keV}$ peak from ${ }^{40} \mathrm{~K}$ in coincidence with the veto, to check the calibration at the lowest energies. To ensure a precise insertion of the copper tube as well as the detector module, a removable frame holding a motorized pulley will be mounted atop the vessel. To further shield the setup from external radiation, the vessel is surrounded by several layers of passive material. The innermost layer is made of polyethylene, with $10 \mathrm{~cm}$ thickness on 


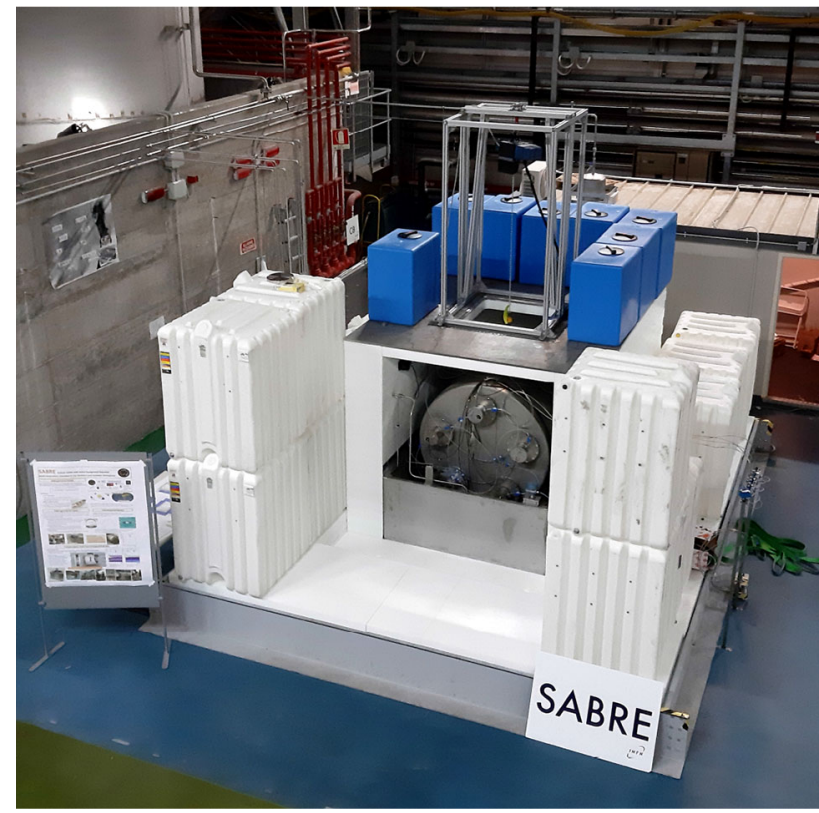

Fig. 3 The SABRE-PoP setup under construction in LNGS Hall-C

the top and bottom and at least $40 \mathrm{~cm}$ on the four sides. The polyethylene supports a $2 \mathrm{~cm}$ steel plate at the top, where the insertion system can be mounted overhanging. Water tanks are placed on top of the plate and on the sides of the shielding for a water thickness of $80 \mathrm{~cm}$ and $91 \mathrm{~cm}$, respectively. The shielding castle is completed with a $15 \mathrm{~cm}$ lead floor. The technical design of the insertion system and of the passive shielding surrounding the vessel is shown in Fig. 2. The polyethylene shell will be sealed, allowing the inner volume to be flushed with dry and clean $\mathrm{N}_{2}$-gas, to avoid radon gas which is present in the laboratory air.

The control of radioactive contamination levels of the detector components (crystals, PMTs, copper enclosure and its parts, vessel, liquid scintillator, etc...), was either performed directly by the SABRE collaboration through radioactivity measurements of various samples, or based on the results of similar screening measurements performed by other experiments for the same material or item, as described in [17] and references therein.

The installation of the Proof-of-Principle setup is currently ongoing at LNGS (see Fig. 3) and data taking is foreseen in 2019.

\section{Sensitivity to the annual modulation}

In this section we study the sensitivity reach of the full-scale SABRE experiment, that will build on the experience and results of the Proof-of-Principle phase. The sensitivity has been studied as a function of the experimental characteristics, such as target mass, measuring time and background level. The results reported in the following assume a total mass of $50 \mathrm{~kg}$ of $\mathrm{NaI}(\mathrm{Tl})$ crystals over 3 years of data collection. We use two different approaches: at first, we study the statistical significance to confirm or reject a signal's modulation with the amplitude reported by DAMA, regardless of any hypothesis on its origin. Later, we draw the standard $90 \%$ CL sensitivity curve for SABRE to spin-independent WIMPnucleon scattering and we show it in comparison with the allowed regions for the DAMA result under these assumptions. We refer to the modulation amplitude reported in [3] for DAMA/NaI and DAMA/LIBRA Phase-I as "the DAMA result" throughout the following.

As the design of the SABRE experiment is not finalized yet, pending the results of the PoP phase, we estimated the background level, as is it customary in our field, using Geant4-based [30] Monte Carlo simulations. These are performed for the PoP setup only and are described in details in [17]. The predicted background level in the energy interval [2-6] $\mathrm{keV}_{\text {ee }}$ for the SABRE-PoP setup is $0.36 \mathrm{cpd} / \mathrm{kg} / \mathrm{keV}$ ee . This is obtained after rejecting all the events accompanied by an energy deposit above a $100 \mathrm{keV}$ threshold in the liquid scintillator veto and under the following assumptions. In the simulation the PoP crystal had the same radio-purity as the $2 \mathrm{~kg}$ test crystal described in Sect. 2.1. Where no measurement was available for the crystal itself, the radio-purity of the Astro Grade powder or, if that was not measured, of the DAMA crystals [24], was assumed.

We find that the background in the energy region of interest for a dark matter search is almost entirely $(90 \%)$ due to the radioactive contamination of the crystal. About $23 \%$ of such background is accounted for by ${ }^{87} \mathrm{Rb},{ }^{238} \mathrm{U}$ and ${ }^{232} \mathrm{Th}$ contaminations, whose values used in the simulation are actually upper limits in the NaI powder, while ${ }^{40} \mathrm{~K}$ contributes with $11 \%$. The ${ }^{210} \mathrm{~Pb}$ is very difficult to predict and cannot be assessed in the grown crystal at the necessary precision prior to the direct underground counting, although the Accelerator Mass Spectroscopy (AMS) technique is being employed to this goal. For the ${ }^{210} \mathrm{~Pb}$ background prediction, we used the upper limit set by DAMA [24] of $0.03 \mathrm{mBq} / \mathrm{kg}$, which gives a $6 \%$ share of the total background. The remaining part is given by cosmogenically-activated isotopes, where ${ }^{3} \mathrm{H}$ accounts alone for about $40 \%$. Cosmogenic activation is calculated using the ACTIVIA [31] simulation software, conservatively estimating an exposure at sea level of about 1 year plus a transport by plane from USA to Italy and only 180 days of underground storage prior to the measurement. The ${ }^{3} \mathrm{H}$ activation calculated for the PoP crystal and assumed in [17] is $0.018 \mathrm{mBq} / \mathrm{kg}$. Transport by ship and longer storage time could actually lead to significant reduction of the cosmogenic activity by a factor $\sim 40 \%$. Concerning the other detector components included in the simulation, as we said, we used radioactive contamination levels either directly measured by the SABRE collaboration or available from the sci- 
entific literature, measured by other experiments for the same material or item. Further details on the background estimates and related simulations can be found in [17,29]. Even though the full scale experiment could achieve lower background level thanks to a larger and improved veto rejection system, we evaluate the SABRE sensitivity using the conservative Proof-of-Principle background estimate.

In order to estimate the sensitivity of SABRE to the DAMA signal, irrespectively of any assumption on its interpretation, we use toy MC simulations of the experimental rate. We generate $50 \mathrm{k}$ data sets that include the expected background and a modulating signal with an amplitude of $0.011 \mathrm{cpd} / \mathrm{kg} / \mathrm{keV}$ ee, which is compatible with both Phase-1 and Phase- 2 results published by DAMA in the [2-6] $\mathrm{keV}_{\text {ee }}$ energy range $[3,4]$. Fitting each data set with a sinusoidal function with a period fixed to 1 year, we obtain a gaussian distribution of the fit amplitudes around the injected value. $90 \%$ of the experiments show a modulation amplitude with a significance of $5.5 \sigma$. We also generate $50 \mathrm{k}$ data sets under a background-only hypothesis. Fitting with the same function, the distribution of the amplitudes centers at zero. $90 \%$ of the simulated experiments show a modulation amplitude incompatible with the DAMA signal at $5 \sigma$.

Next, we evaluate the sensitivity of SABRE to spinindependent WIMP nuclear scattering. The expected dark matter interaction rate on a $\mathrm{NaI}$ target has been evaluated as a function of time using the expression given in [12]. This assumes the standard WIMP halo model and a spinindependent WIMP nucleon interaction. We also assume an average Earth velocity of $232 \mathrm{~km} / \mathrm{s}$, a Galaxy escape velocity of $544 \mathrm{~km} / \mathrm{s}$, and that the WIMP velocity follows a Maxwellian distribution with most probable speed of 220 $\mathrm{km} / \mathrm{s}$. Concerning the quenching factor of nuclear recoils on sodium, we applied the energy dependent measurements from [32]. As for iodine we assumed the value of 0.09 , also used by DAMA [33]. The detection efficiency and resolution were set at the values reported by the DAMA collaboration [24] for their detectors.

The sensitivity curve for SABRE was calculated as follows. For several combinations of WIMP mass $\left(M_{W}\right)$ between 1 and $1000 \mathrm{GeV}$ and cross section $\left(\sigma_{S I}\right)$ between $10^{-42}$ and $10^{-36} \mathrm{~cm}^{2}$, we generate 1000 data sets binned in 30-day intervals, obtained with a Poisson extraction that accounts for the expected unmodulated rate plus the background from Monte Carlo. This process is performed by varying the energy from 2 to $6 \mathrm{keV}$ in eight $0.5 \mathrm{keV}$ bins. We fit each data set with a cosine function having a 1-year period and we obtain a zero-centered gaussian distribution of amplitudes. We then sum over the eight bins the $\chi^{2}$ of the modulation amplitude function with respect to the nonmodulating data set, considering the standard deviation of the respective gaussian distribution of amplitudes as the sigma of each bin. The resulting $\chi^{2}\left(M_{W}, \sigma_{S I}\right)$ is then cut at $90 \%$ $\mathrm{CL}$ and the sensitivity curve is obtained.

The dominant sources of systematic uncertainty for the calculation of the sensitivity curve are the energy resolution, the detection efficiency, the sodium and the iodine quenching factors, and the background level. For the energy resolution we have used the same parameterization reported by DAMA in [24] $\sigma(E) / E=0.0091+0.488 / \sqrt{E}$, assuming a gaussian uncertainty of $25 \%$ on the coefficients. The detector efficiency is estimated taking the average of the values reported by DAMA for their Phase- 1 and Phase- 2 data [34], and assuming the difference of the two as a gaussian uncertainty. For the sodium quenching factor we have fit the measurements of [32] as a function of the recoil energy with a linear function, using the uncertainties of the fit as our systematics. The uncertainty that we assumed for iodine quenching factor is 0.01 , following the analysis described in [35]. Finally, for the background level, we have assumed a conservative systematic uncertainty of $30 \%$. The systematics associated with the choice of the energy binning was found to be negligible around our choice of $0.5 \mathrm{keV}_{\mathrm{ee}}$. All of the above parameters have been simultaneously varied by randomly sampling 1000 times from their expected distributions. We used each set of sampled values to calculate 1000 expected annually modulated daily rates, fit with a cosine function, build the distribution of sensitivity values as described above, and extract the $90 \%$ CL sensitivity as the median of that distribution (black solid line) and the $1 \sigma$ (green) and $2 \sigma$ (yellow) regions shown in Fig. 4. The systematic on the sodium quenching factor is relevant only for WIMP masses below $20 \mathrm{GeV}$, while that on iodine quenching factor has the bigger impact on WIMP masses from 15 to $60 \mathrm{GeV}$ and above $150 \mathrm{GeV}$. The efficiency and background systematics give a significant contribution for all WIMP masses, while the resolution effect is practically negligible.

The experiment is sensitive to spin-independent WIMPnucleon scattering cross sections as small as $2 \times 10^{-42} \mathrm{~cm}^{2}$ for a WIMP mass of 40-50 GeV.

We have added to the plot the 3- and 5-sigma confidence regions we obtained interpreting the DAMA Phase-1 results $[3,36]$ in the framework of the standard WIMP model, as described in [37] under the same assumptions on WIMP interaction, halo model, quenching factor, energy resolution and detection efficiency. There are two caveat on this plot; the first is that, as stated, the comparison is model-dependent. The second is that the interpretation of DAMA Phase-2 results with a $1 \mathrm{keV}_{\mathrm{ee}}$ energy threshold [4] seems disfavoured in the framework of the standard WIMP model. Other theoretical explanations are being proposed $[34,38,39]$. We have also produced similar plots using different assumptions for the sodium quenching factor, given that several independent measurements are available $[32,33,40]$ and verified that the 


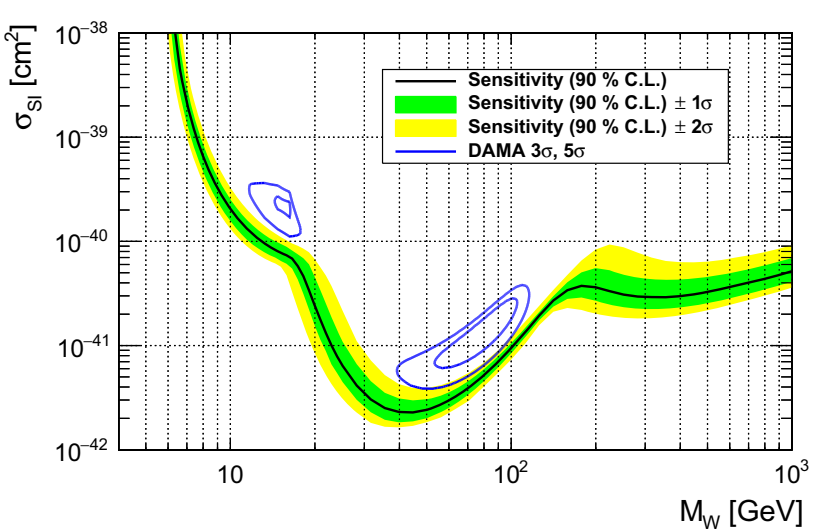

Fig. 4 SABRE sensitivity for a total mass of $50 \mathrm{~kg}$ of $\mathrm{NaI}(\mathrm{Tl})$ crystals over 3 years of data collection, with uncertainty bands that cover different modeling of efficiency and energy resolution. The blue curves represent the 3 and 5 sigma confidence regions we obtained interpreting the DAMA Phase-1 results $[3,36]$ in the framework of the standard WIMP model, as described in [37]

relative position of our sensitivity curve and the lower energy DAMA-allowed region is independent of this parameter.

\section{Conclusions}

The SABRE experiment will investigate the expected annual modulation due to interaction of dark matter particles in the galactic halo on $\mathrm{NaI}(\mathrm{Tl})$ scintillating crystals. SABRE aims to achieve a background at the level of $0.1 \mathrm{cpd} / \mathrm{kg} / \mathrm{keV}_{\text {ee }}$ via crystal purity and active rejection through a liquid scintillator veto. The experiment also adopts PMTs with high quantumefficiency and low background, which are directly coupled to the crystals, to maximize the light collection efficiency and gain sensitivity for energies below $2 \mathrm{keV}_{\text {ee }}$. SABRE foresees the installation of twin detectors underground at LNGS in Italy and at SUPL (Stawell Underground Physics Laboratory) in Australia. The dual location in opposite hemispheres allows the identification of any local and/or seasonal effects that could possibly contribute a modulation of the experimental rate. If the assumed conditions are achieved, SABRE will be the highest sensitivity $\mathrm{NaI}(\mathrm{Tl})$ based experiment and it will be able to either verify or refute, in a model-independent way, the long debated result of the DAMA experiment. Under the assumption of a spin-independent WIMP-nucleus interaction, after 3 years of exposure and with a total mass of $50 \mathrm{~kg}$, the experiment is expected to be sensitive to WIMPnucleon scattering cross sections down to $2 \times 10^{-42} \mathrm{~cm}^{2}$ for a WIMP mass of 40-50 GeV.

Acknowledgements The SABRE program is supported by funding from INFN (Italy), NSF (USA), and ARC (Australia Grants: LE170100162, LE16010080, DP170101675, LP150100075). F. Froborg has received funding from the European Union's Horizon 2020 research and innovation programme under the Marie Sklodowska-Curie grant agreement No 703650. We acknowledge the generous hospitality and constant support of the Laboratori Nazionali del Gran Sasso (Italy).

Data Availability Statement This manuscript has no associated data or the data will not be deposited. [Authors' comment: This paper describes the concept, the design and the physics reach of a new experiment. No experimental data were used in the present study.]

Open Access This article is distributed under the terms of the Creative Commons Attribution 4.0 International License (http://creativecomm ons.org/licenses/by/4.0/), which permits unrestricted use, distribution, and reproduction in any medium, provided you give appropriate credit to the original author(s) and the source, provide a link to the Creative Commons license, and indicate if changes were made. Funded by $\mathrm{SCOAP}^{3}$.

\section{References}

1. A.K. Drukier, K. Freese, D.N. Spergel, Phys. Rev. D 33, 3495 (1986). https://doi.org/10.1103/PhysRevD.33.3495

2. K. Freese, J. Frieman, A. Gould, Phys. Rev. D 37, 3388 (1988). https://doi.org/10.1103/PhysRevD.37.3388

3. R. Bernabei et al., Eur. Phys. J. C 73, 2648 (2013). https://doi.org/ 10.1140/epjc/s10052-013-2648-7

4. R. Bernabei et al., Nucl. Phys. Atom. Energy 19(4), 307 (2019). https://doi.org/10.15407/jnpae2018.04.307

5. E. Aprile et al., Phys. Rev. Lett. 121, 111302 (2018). https://doi. org/10.1103/PhysRevLett.121.111302

6. P. Agnes et al., Phys. Rev. Lett. 121, 081307 (2018). https://doi. org/10.1103/PhysRevLett.121.081307

7. R. Agnese et al., Phys. Rev. D 97, 022002 (2018). https://doi.org/ 10.1103/PhysRevD.97.022002

8. A.H. Abdelhameed, et al., arXiv:1904.00498 (2019)

9. P. Adhikari et al., Nature 564(7734), 83 (2018). https://doi.org/10. 1038/s41586-018-0739-1

10. I. Coarasa, et al., arXiv:1812.02000 (2018)

11. V. Gluscevic et al., JCAP 1512(12), 057 (2015). https://doi.org/10. 1088/1475-7516/2015/12/057

12. K. Freese, M. Lisanti, C. Savage, Rev. Mod. Phys. 85, 1561 (2013). https://doi.org/10.1103/RevModPhys.85.1561

13. S.K. Lee et al., Phys. Rev. Lett. 112(1), 011301 (2014). https://doi. org/10.1103/PhysRevLett.112.011301

14. J. Amare et al., arXiv:1812.01377 (2018)

15. P. Adhikari et al., EPJC 78(6), 490 (2018). https://doi.org/10.1140/ epjc/s10052-018-5970-2

16. J. Amaré et al., Eur. Phys. J. C 76(8), 429 (2016). https://doi.org/ 10.1140/epjc/s10052-016-4279-2

17. M. Antonello et al., Astropart. Phys. 106, 1 (2019). https://doi.org/ 10.1016/j.astropartphys.2018.10.005

18. Sigma Aldrich, http://www.sigmaaldrich.com/united-states.html. Accessed 25 June 2018

19. PNNL, Private communication

20. RMD, Inc., http://rmdinc.com/. Accessed 25 June 2018

21. P.W. Bridgman, Proc. Am. Acad. Arts Sci. 60(6), 305 (1925)

22. I.J. Arnquist, E.W. Hoppe, Nucl. Instrum. Methods A 851, 15 (2017). https://doi.org/10.1016/j.nima.2017.01.064

23. Seastar Chemicals. Private communication

24. R. Bernabei et al., Nucl. Instrum. Methods A 592(3), 297 (2008). https://doi.org/10.1016/j.nima.2008.04.082

25. J. Amare et al., In: 15th International conference on topics in astroparticle and underground physics (TAUP 2017) Sudbury, Ontario, Canada, July 24-28, 2017 (2017) 
26. J.S. Park et al., Nucl. Instrum. Methods A851, 103 (2017). https:// doi.org/10.1016/j.nima.2017.01.041

27. E. Aprile et al., Eur. Phys. J. C 75(11), 546 (2015). https://doi.org/ 10.1140/epjc/s10052-015-3657-5

28. G. Alimonti et al., Nucl. Instrum. Methods A 609(1), 58 (2009). https://doi.org/10.1016/j.nima.2009.07.028

29. E. Shields, SABRE: a search for dark matter and a test of the DAMA/LIBRA annual-modulation result using thallium-doped sodium-iodide scintillation detectors. Ph.D. thesis, Princeton University (2015)

30. S. Agostinelli et al., Nucl. Instrum. Methods A 506(3), 250 (2003). https://doi.org/10.1016/S0168-9002(03)01368-8

31. ACTIVIA Simulation Software, http://universityofwarwick. github.io/ACTIVIA/. Accessed 18 April 2019

32. J. Xu et al., Phys. Rev. C 92(1), 015807 (2015). https://doi.org/10. 1103/PhysRevC.92.015807
33. R. Bernabei et al., Phys. Lett. B 389(4), 757 (1996). https://doi. org/10.1016/S0370-2693(96)80020-7

34. S. Baum et al., Phys. Lett. B 789, 262 (2019). https://doi.org/10. 1016/j.physletb.2018.12.036

35. V.I. Tretyak, Astropart. Phys. 33, 40 (2010)

36. R. Bernabei et al., Int. J. Mod. Phys. A 28(16), 1330022 (2013). https://doi.org/10.1142/S0217751X13300226

37. C. Savage et al., J. Cosmol. Astropart. Phys. 2009(04), 010 (2009)

38. S. Kang et al., J. Cosmol. Astropart. Phys. 2018(07), 016 (2018). https://doi.org/10.1088/1475-7516/2018/07/016

39. J. Herrero-Garcia et al., Phys. Rev. D 98, 123007 (2018). https:// doi.org/10.1103/PhysRevD.98.123007

40. T. Stiegler et al., arXiv:1706.07494 (2017) 